\title{
Cartographie Des Risques De L'erosion Hydrique Par L'equation Universelle Revisee Des Pertes En Sols, La Teledetection Et Les Sig Dans Le Bassin Versant De L’ourika (Haut Atlas, Maroc)
}

\author{
Meliho Modeste \\ Université Mohamed V, Institut Scientifique Rabat, Maroc \\ Khattabi Abdellatif \\ Ecole Nationale Forestière d’Ingénieurs Salé, Maroc \\ Mhammdi Nadia \\ Université Mohamed V, Institut Scientifique Rabat, Maroc \\ Hongming Zhang \\ Northwest A \& F University, Yangling, Shaanxi, China
}

doi: 10.19044/esj.2016.v12n32p277 URL:http://dx.doi.org/10.19044/esj.2016.v12n32p277

\begin{abstract}
Covering an area of 576 square kilometers, the Ourika watershed is a sub-watershed of the large Tensift basin, located on the northwest slopes of the Marrakech High Atlas, in mid-west Morocco. This basin of dramatic topography, with sparse vegetation cover and friable substrates, is under increasing human action exacerbated by a variable and changing climate. Its vulnerability to water erosion is quite high, increasing risks of wadi flows with significant sediment loads. The aim of this work was to quantify soil loss in the basin using the Revised Universal Soil Loss Equation (RUSLE) and Geographic Information System. The results showed that the Ourika watershed was subject to strong climatic aggressiveness ranging from 55.22 to $100.57 \mathrm{MJ} . \mathrm{mm} / \mathrm{ha}$.h. The average soil erodibility value, $\mathrm{K}$, was 0.48 t.ha.h/ha.MJ.mm, with a standard deviation of 0.28 t.ha.h/ha.MJ.mm. Slopes with values higher than $35 \%$ represented $72 \%$ of the watershed's area, with the topographic factor, LS, values ranging from 0.01 to 94.5 . The vegetation factor was high throughout the Ourika watershed while $C$ values were higher than 0.5 for $73 \%$ of the watershed's area. The average soil loss obtained in the basin was $380 \mathrm{t} / \mathrm{ha} /$ year. These results indicated that $48 \%$ of the watershed's area was subject to a soil loss between 50-400 t/ha/year, and between 400 and $1000 \mathrm{t} / \mathrm{ha} /$ year for $30 \%$ of the watershed. Soil loss below the tolerance level ( $<7 \mathrm{t} / \mathrm{ha} /$ year) represented only $4 \%$ of the watershed area.
\end{abstract}


These findings served in highlighting the significance of erosion in the Ourika watershed.

Keywords: Water erosion, RUSLE, Geographic Information System, Ourika watershed, Morocco

\section{Résumé}

D’une superficie de $576 \mathrm{Km}^{2}$, le bassin versant de l'Ourika est un sous bassin du grand bassin de Tensift qui se situe sur le versant nord-ouest du Haut Atlas de Marrakech, dans le centre-Ouest du Maroc. Ce bassin d'une topographie très accidentée, à couvert végétal peu dense et à substrats friables, est soumis à une action anthropique croissante exacerbée par un climat variable et changeant. Sa vulnérabilité aux phénomènes d’érosion hydrique est assez élevée engendrant ainsi un risque d'écoulements des oueds avec des charges solides importantes. L'objectif de ce travail est de quantifier les pertes en sols dans ce bassin en utilisant l'Equation Universelle Révisée des Pertes en sols (RUSLE) et les Systèmes d’Information Géographique. Les résultats obtenus révèlent que le bassin versant de l'Ourika est soumis à une forte agressivité climatique variant de 55,22 à 100,57 MJ.mm/ha.h. La valeur moyenne de l'érodibilité des sols $\mathrm{K}$ est de 0,48 t.ha.h/ha.MJ.mm avec un écart type de 0,28 t.ha.h/ha.MJ.mm. Les pentes supérieures à 35\% représentent $72 \%$ de la superficie du bassin et les valeurs du facteur topographique LS varient de 0,01 à 94,5. Le facteur couvert végétal est élevé dans le bassin versant de l’Ourika ; les valeurs de C sont supérieures à 0,5 pour $73 \%$ de la superficie du bassin. La valeur moyenne des pertes en sols (t/ha/an) obtenue dans le bassin est de 380 t/ha/an. Les résultats obtenus indiquent que $48 \%$ de la superficie du bassin est soumis à des pertes en sols comprises entre 50-400 t/ha/an, et 30\% de la superficie du bassin à des pertes variant entre 400 et 1000 t/ha/an. Les pertes en sols au-dessous du seuil de tolérance ( $<7 \mathrm{t} / \mathrm{ha} / \mathrm{an})$ ne représentent que 4\% de la superficie du bassin versant. Ces résultats témoignent de l'importance des phénomènes d’érosion dans le bassin versant de l’Ourika.

Mots clés : Erosion hydrique, RUSLE, Système d'Information Géographique, Bassin versant de l’Ourika, Maroc

\section{Introduction}

L'érosion des sols associée à la dégradation des terres sont des phénomènes spatio-temporels qui prennent de l'ampleur dans plusieurs pays du monde (Hoyos, 2005 ; Pandey et al., 2009). L'érosion des sols est un phénomène naturel inévitable qui devient un sérieux problème environnemental et économique quand il est accéléré par les activités 
humaines (Lal, 1998 ; Del Mar López et al, 1998). Les phénomènes d’érosion hydrique se sont accélérés partout dans le monde (De Graaf, 1996). La pression démographique et l'extension des cultures par diverses activités humaines telles que les pratiques agricoles, les exploitations forestières, les pâturages, la construction des routes et bâtiments; associées aux effets amplificateurs des changements climatiques, ont conduit à l'exposition des terres au ruissellement, et ainsi, à la dégradation des sols par l'érosion hydrique (Vezena and Bonn, 2006) ; Boudhar et al., 2007). Ainsi, l’érosion hydrique affecte la qualité des sols en induisant la détérioration de leurs couches superficielles riches en matière organique. Ainsi, l'érosion des sols conduit à la perte de productivité des sols et à la dégradation des terres.

Le Haut Atlas est la chaîne la plus élevée du Maroc, englobant le mont Toubkal culminant à $4167 \mathrm{~m}$. Cette chaine montagneuse est structurée en une multitude de bassins versants qui constituent un véritable château d'eau pour les plaines arides avoisinantes. Cependant, les différentes zones du Haut Atlas sont sujettes à de fortes contraintes qui affectent négativement leur fonctionnement hydrologique et font de ces massifs montagneux un milieu naturel particulièrement sensible aux actions anthropiques (Meliho et al., 2016). Parmi ces contraintes, on cite particulièrement une topographie très accidentée, des sols pauvres en matière organique et faiblement épais, des roches mères imperméables, des substrats friables, un climat rude et parfois brutal, et une couverture végétale souvent peu dense, ce qui le rend très exposé au phénomène d’érosion (Meliho et al., 2016).

La quantification de l'érosion hydrique est normalement effectuée en utilisant des modèles adaptés au but recherché. Les modèles mathématiques utilisés dans cette quantification sont continuellement améliorés par les chercheurs dans beaucoup de pays en les adaptant aux conditions locales (Arnalds et al., 2001). Plusieurs modèles sont actuellement développés et utilisés dans plusieurs pays et dans différentes régions. Les modèles de prédiction d'érosion aident les décideurs dans la planification de la gestion des milieux naturels et agricoles (Angima et al., 2003). Bien qu'il soit difficile de trouver un modèle qui considère toutes les formes d'érosion, certains modèles ont été développés pour aider les gestionnaires des eaux et des sols à identifier les zones les plus vulnérables à l'érosion. Le modèle RUSLE (Renard et al., 1997) est largement utilisé dans la région méditerranéenne. C'est un modèle empirique basé sur le modèle USLE (Wischmeier and Smith, 1978). L'utilisation de la télédétection et des systèmes d'information géographique a rendu possible l'estimation et la spatialisation de l'érosion des sols à un coût raisonnable (Boggs et al., 2001 ; Wilson and Lorang, 2000; Wang et al., 2003 ; Jasrotia and Singh, 2006). Le principe consiste à intégrer dans un système d'information géographique les facteurs figurant dans le modèle empirique RUSLE. 
L'objectif de ce travail est d'analyser et spatialiser des différents facteurs intervenant dans le phénomène de l'érosion et d'aboutir à la carte des risques d'érosion et des pertes en sols dans le bassin versant de l'Ourika. La cartographie des facteurs d'érosion du sol et l'identification des zones vulnérables, permettraient d'évaluer les risques d'érosion en vue d'envisager des mesures de conservation des sols et de gestion des ressources hydriques.

\section{Matériels et méthodes}

\section{Zone d'étude}

Le bassin versant de l'Ourika se situe dans le Haut Atlas de Marrakech, Maroc. Il constitue un sous bassin atlasique du grand bassin versant du Tensift (Fig 1). Ce bassin couvre une superficie de $576 \mathrm{~km}^{2}$. Il est situé entre les latitudes $31^{\circ}$ et $31^{\circ} 21^{\prime}$ Nord et les longitudes $7^{\circ} 30^{\prime}$ et $7^{\circ} 60^{\prime}$ Ouest. Il est limité au Sud par le haut bassin de l'Oued Souss, au Nord par la plaine du Haouz, à l'Est par le bassin versant de Zat et à l'ouest par le bassin versant de la Rhéraya.

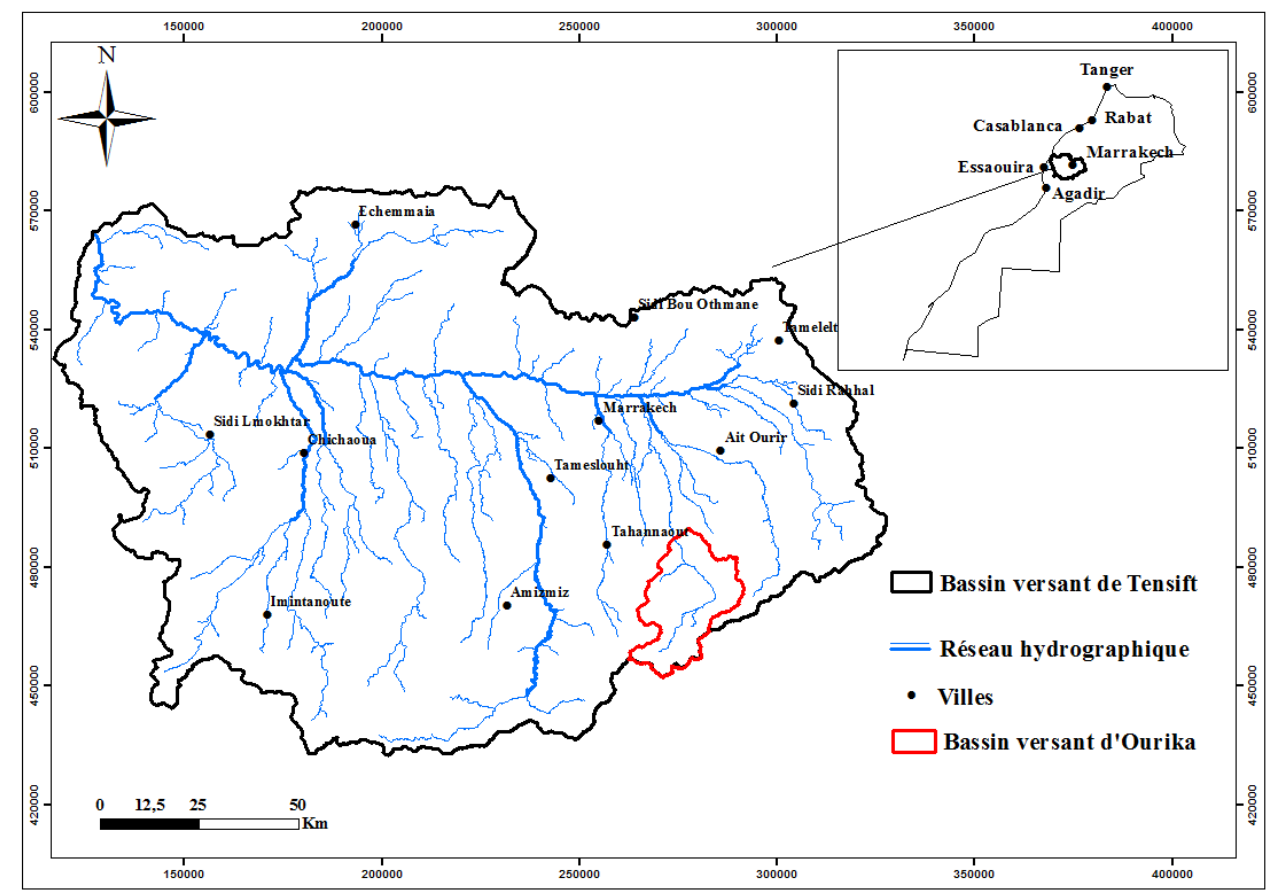

Fig 1. Situation géographique du bassin versant de l'Ourika

La zone est caractérisée par trois principales unités physiographiques : les zones du piémont avec une altitude aux alentours de $600 \mathrm{~m}$, les vallées de l'Oued Ourika et ses affluents et les zones de haute montagne et les plateaux d'altitude. 
Sur le plan topographique, l’altitude moyenne s’élève à 2500 m avec prédominance de terrains compris entre 1600 et 3200 m (75\%).

Les pentes du bassin sont dans l'ensemble fortes, amplifiant ainsi les phénomènes de ruissellement et d'érosion.

Sur le plan géologique, le bassin versant de l'Ourika offre deux grands types de faciès (Biron, 1982): un substrat rocheux dur (roches éruptives ou métamorphiques) situé dans la partie amont du bassin versant représentant environ $61 \%$ de son étendue et un substrat tendre à moyennement tendre situé à des altitudes inférieures, composé de dépôts permo-triasiques et quaternaires, représentant environ $39 \%$ de la superficie du bassin. Il s’agit des grès friables et marnes rouges, des conglomérats et grès rouges massifs, et des flysch schisto-grèseux.

Les ravins de la partie amont traversent un substrat rocheux dur caractérisé principalement par de faibles apports solides. Les eaux au moment des crues sont claires et non chargées. Les ravins situés dans la partie centrale du bassin sont par contre très vulnérables à l'érosion hydrique et charrient par conséquent des apports solides et liquides importants.

Le climat du bassin versant de l’Ourika est caractérisé par sa grande variabilité spatiotemporelle. La précipitation annuelle est en moyenne de 500 mm. Cette pluviométrie augmente avec l'altitude. Elle est de l'ordre de 400 $\mathrm{mm}$ au piedmont et peut dépasser $700 \mathrm{~mm}$ par an sur les hauts sommets du bassin. Les températures moyennes varient de 21.5 à $32^{\circ} \mathrm{C}$ pour les maximas et de 4 à $5.7^{\circ} \mathrm{C}$ pour les minimas. Les mois de juillet et août sont les mois les plus chauds alors que les mois de décembre et janvier sont les mois les plus froids de l'année

Le couvert végétal au niveau de la zone se trouve en grande partie dans l'étage Oroméditerranéen à base de matorrals de xérophytes épineuses et d'hémicryptophytes d'altitude (Ouhammou, 1986). La végétation arborée au niveau du bassin versant, est représentée par les étages Méditerranéen, Mésoméditerranéen et Thermoméditerranéen. Il s’agit essentiellement de forêts de chêne vert, de genévriers, et de thuya (Ouhammou, 1986).

\section{Méthodologie}

Le modèle le plus utilisé pour la quantification des pertes en sols est le modèle USLE avec sa version révisée RUSLE grâce à la facilité de son application et à sa compatibilité avec les systèmes d'information géographiques (Millward and Mersey, 1999 ; Lu et al., 2004 ; Dabral et al., 2008 ; Pandey et al., 2009; Bonilla et al., 2010). Le modèle RUSLE a été largement utilisé aussi bien dans les milieux agricoles que forestiers pour la prédiction et la distribution spatiale de la moyenne annuelle des pertes en sols en intégrant les différents facteurs d'érosion (Wischmeier and Smith, 1978 ; Renard et al., 1997). L’équation s’exprime par la relation : 
$\mathrm{A}=\mathrm{R} * \mathrm{~K} * \mathrm{LS} * \mathrm{C} * \mathrm{P}$

Où :

$\mathrm{A}=$ taux de perte en sol (t/ha/an),

$\mathrm{R}=$ érosivité des pluies (MJ.mm/ ha.h.an),

$\mathrm{K}$ = érodibilité du sol (t.h/ha. MJ.mm),

$\mathrm{LS}=$ facteur topographique ( $\mathrm{L}$ en $\mathrm{m}, \mathrm{S}$ en \%),

$\mathrm{C}=$ facteur de couverture végétale,

$\mathrm{P}=$ facteur des pratiques agricoles.

\section{Facteur d'érosivité des pluies (R)}

L'érosivité est la capacité potentielle de la pluie à produire l'érosion (Hudson, 1981). C’est le produit de l'énergie cinétique des pluies et de l'intensité maximale des pluies durant 30 minutes (Wischmeier and Smith, 1978). Elle peut aussi être considérée comme l'indice moyen annuel d'érosion par la pluie. Du fait que les données d'énergie cinétique et d'intensité des pluies sont difficiles à obtenir, des formules alternatives ne nécessitant que des données mensuelles et annuelles pour la détermination du facteur R ont été développées (Kalman, 1967 ; Arnoldus, 1980 ; Rango and Arnoldus, 1987). Pour estimer ce facteur, nous avons appliqué la formule de Rango \& Arnoldus (Rango and Arnoldus, 1987) à 5 stations se trouvant dans le bassin versant de l'Ourika sur une période de 20 ans (1995-2015). Une période de 20 à 25 ans est recommandée pour le calcul de la valeur moyenne de R au niveau d'une station (Millward and Mersey, 1999). L'interpolation spatiale du facteur $\mathrm{R}$ a été effectuée par krigeage en utilisant l'outil de géospatialisation d'ArcGIS.

$\log \mathrm{R}=1,74 * \log \sum\left(\mathrm{P}_{\mathrm{i}}^{2} / \mathrm{P}\right)+1,29$

Où :

$\mathrm{Pi}$ : précipitations mensuelles

$\mathrm{P}$ : précipitations annuelles en $\mathrm{mm}$.

\section{Facteur d'érodibilité des sols (K)}

Le facteur d'érodibilité (K) des sols exprime la cohésion et la résistance du sol vis-à-vis de l'érosion. Il est fonction de la texture du sol, de sa structure, de son taux de matière organique et de sa perméabilité.

Les expérimentations sur différents types de sols ont permis de développer statistiquement une équation pour le calcul de ce facteur (Wischmeier and Smith, 1978) :

$\mathrm{K}=1 / 100 *\left[2,1 * \mathrm{M}^{1,14} * 10^{-4}(12-\mathrm{A})+3,25(\mathrm{~B}-2)+2,5(\mathrm{C}-3)\right]$

Où :

$\mathrm{M}=(\%$ sable fin $+\%$ limon $) *(100-\%$ argile $)$

$\mathrm{A}=$ pourcentage de matière organique

$\mathrm{B}$ = code de la perméabilité 
$\mathrm{C}=$ code de la structure

A défaut de la disponibilité d'une carte pédologique de la zone d'étude, nous avons utilisé la carte des substrats géologiques et celle de l'occupation du sol pour identifier des unités homogènes. Des prélèvements de sols ont été ensuite effectués dans ces unités (17 prélèvements) et analysés au laboratoire pour la détermination du facteur d’érodibilité K.

\section{Facteur topographique (LS)}

Le modèle RUSLE prend en compte la longueur et l'inclinaison des pentes qui affectent la production et le transport de sédiments dans un site. D’autres facteurs tels que la compaction et les perturbations du sol sont aussi considérés dans la génération du facteur LS (Prasannakumar et al., 2012). L'érosion augmente avec l'inclinaison de la pente mais, contrairement au facteur L qui exprime l'effet de la longueur de la pente, le modèle RUSLE ne fait aucune différence entre l'érosion en nappe et l'érosion linéaire dans le facteur S qui exprime l'effet de l'inclinaison de la pente sur l'érosion des sols (Renard et al., 1997; Lu et al., 2004 ; Krishna Bahadur, 2009).

Le facteur LS a été calculé par l’outil de facteur LS (LS-TOOL) développé par Zhang et al (Zhang et al, 2013). La méthodologie de calcul du facteur LS est appliquée à chaque pixel du modèle numérique d'altitude. Le calcul du facteur L est basé sur l'équation développée par Desmet et Govers (Desmet and Govers, 1996). Le calcul du facteur S est basé sur l'équation développée par Wischmeier et Smith (Wischmeier and Smith, 1978) et modifiée dans le modèle RUSLE pour une meilleure représentation du degré d’inclinaison des pentes (McCool et al., 1987).

Dans le souci de la précision du facteur LS qui dépend de la résolution du MNT (Molnar and Julien, 1998 ; Panagos et al., 2015), un MNT de résolution $10 \mathrm{~m}$ a été utilisé pour le calcul du facteur LS.

\section{Facteur de couverture végétale $(C)$}

Un sol bien protégé par une couverture végétale réduit considérablement l'effet des facteurs d'agressivité climatique, d’érodibilité du sol, de l'inclinaison du degré de la pente, quel que soit leur importance. En ce sens, un sol bien couvert réduit le ruissellement en facilitant l'infiltration et empêchant le déclenchement de l'érosion.

Le facteur $\mathrm{C}$ compare les pertes en sol liées à une terre bien couverte par une végétation naturelle dense, celles liées à une terre nue sans aucun couvert végétal et celles soumises à une gestion spécifique (Wischmeier and Smith, 1978). Il varie de 0,001 sous forêt dense à 1 sur un sol nu. Les données de télédétection sont utilisées pour le calcul du facteur C (Karydas et al., 2009 ; Tian et al., 2009). La formule utilisée pour la détermination du facteur C (Zhou et al., 2008 ; Kouli et al., 2009) en utilisant l'indice de 
végétation normalisé NDVI renseignant sur le couvert végétal et sa santé (Jensen, 2000) est :

\section{$\mathrm{C}=\exp [-(\alpha \mathrm{NDVI} /(\boldsymbol{\beta}-\mathrm{NDVI}))]$}

Où $\alpha$ et $\beta$ sont des paramètres sans unités qui déterminent la forme de la courbe reliant le NDVI au facteur C. Ces auteurs ont attribué les valeurs 2 et 1 respectivement à $\alpha$ et $\beta C$ ette équation est plus précise qu'une relation linéaire (Van der Knijff et al., 2000) et a été utilisée avec succès dans les mêmes conditions pédologiques et climatiques (Markhi et al., 2015).

\section{Facteur pratiques antiérosives $\mathbf{P}$}

Les pratiques culturales comme le labour selon les courbes de niveau, cultures en bandes alternées ou en terrasses, le billonnage etc., sont des pratiques efficaces de conservation des sols. Les valeurs de $\mathrm{P}$ ont été estimées en fonction de la pente (Shin, 1999), selon le tableau 1.

Tableau 1. Valeur du facteur lié aux pratiques antiérosives en fonction de la pente (Shin, 1999).

\begin{tabular}{|c|c|}
\hline Pente (\%) & P \\
\hline $0.0-7.0$ & 0.55 \\
\hline $7.0-11.3$ & 0.60 \\
\hline $11.3-17.6$ & 0.80 \\
\hline-26.8 & 0.90 \\
\hline$>26.8$ & 1.00 \\
\hline
\end{tabular}

\section{Résultats et discussion}

Pour estimer les pertes en sols dans le bassin versant de l'Ourika, le modèle RUSLE a été utilisé. Les différents facteurs du modèle ont été estimés et spatialisés dans un environnement SIG. La combinaison de ces différents facteurs a permis d’obtenir la carte de risques d'érosion du bassin.

\section{Facteur d'érosivité de pluie (R)}

L’érosivité des pluies dans le bassin versant de l'Ourika varie de 55 à 100 MJ. mm/ha.h.an (Fig 2). L'agressivité pluviale connait un gradient croissant du Nord-Est du bassin vers le Sud-Ouest. 


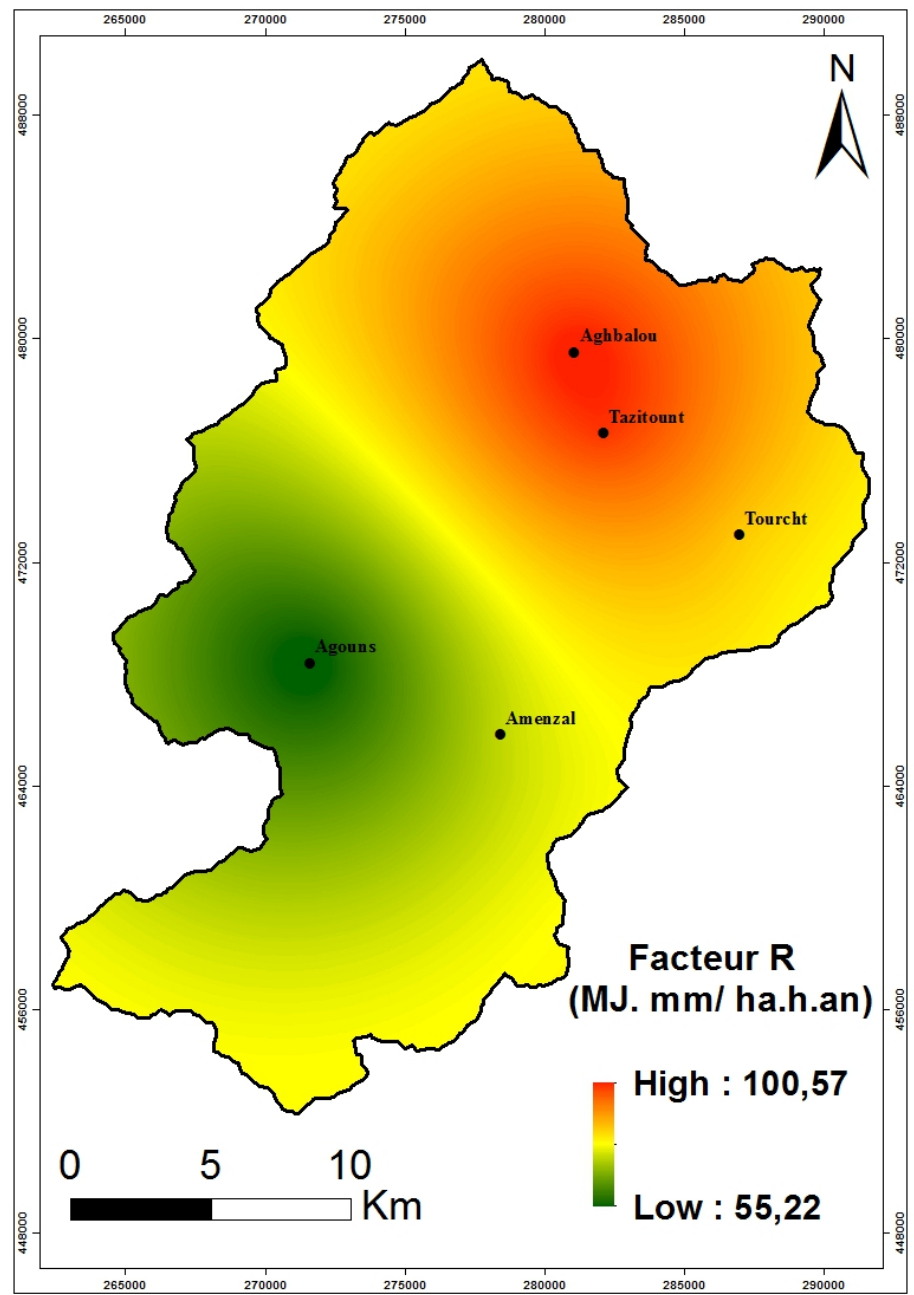

Fig 2. Carte de l'érosivité des pluies (R) dans le bassin versant de l'Ourika

Ces valeurs de $\mathrm{R}$ dépassant 50 MJ. mm/ha.h.an indiquent que la totalité du bassin versant de l'Ourika est soumise à une agressivité climatique élevée. On en déduit donc que le pouvoir érosif des pluies est important dans ce bassin. Les valeurs déduites sont plus importantes que celles obtenues dans le bassin versant de N'fis (41.40 à 57.28 MJ. $\mathrm{mm} /$ ha.h.an), un bassin séparé du bassin de l'Ourika par le bassin de Rhéraya (Markhi et al., 2015), celles obtenues dans le bassin de Boussouab (31,2 à 60 MJ. mm/ha.h.an) dans la partie orientale du Rif (Sadiki et al., 2004) et celles obtenues dans le Rif central avec des valeurs variant de 43 à 87,56 MJ. $\mathrm{mm} /$ ha.h.an (Rahhou, 1999). Par contre, ces valeurs sont inférieures à celles obtenues dans le bassin d'Oum Er-Rbia, comprises entre 70 et 119 MJ. $\mathrm{mm} /$ ha.h.an (Yjjou et al., 2014) et celles obtenues dans le bassin de Telata allant de 215 et 228 MJ. mm/ha.h.an (Merzouk and Dahman, 1998). 


\section{Facteur d'érodibilité des sols (K)}

Le facteur $\mathrm{K}$ a été déterminé par analyses des échantillons de sols en utilisant la relation de Wishmeier et Smith (1978).

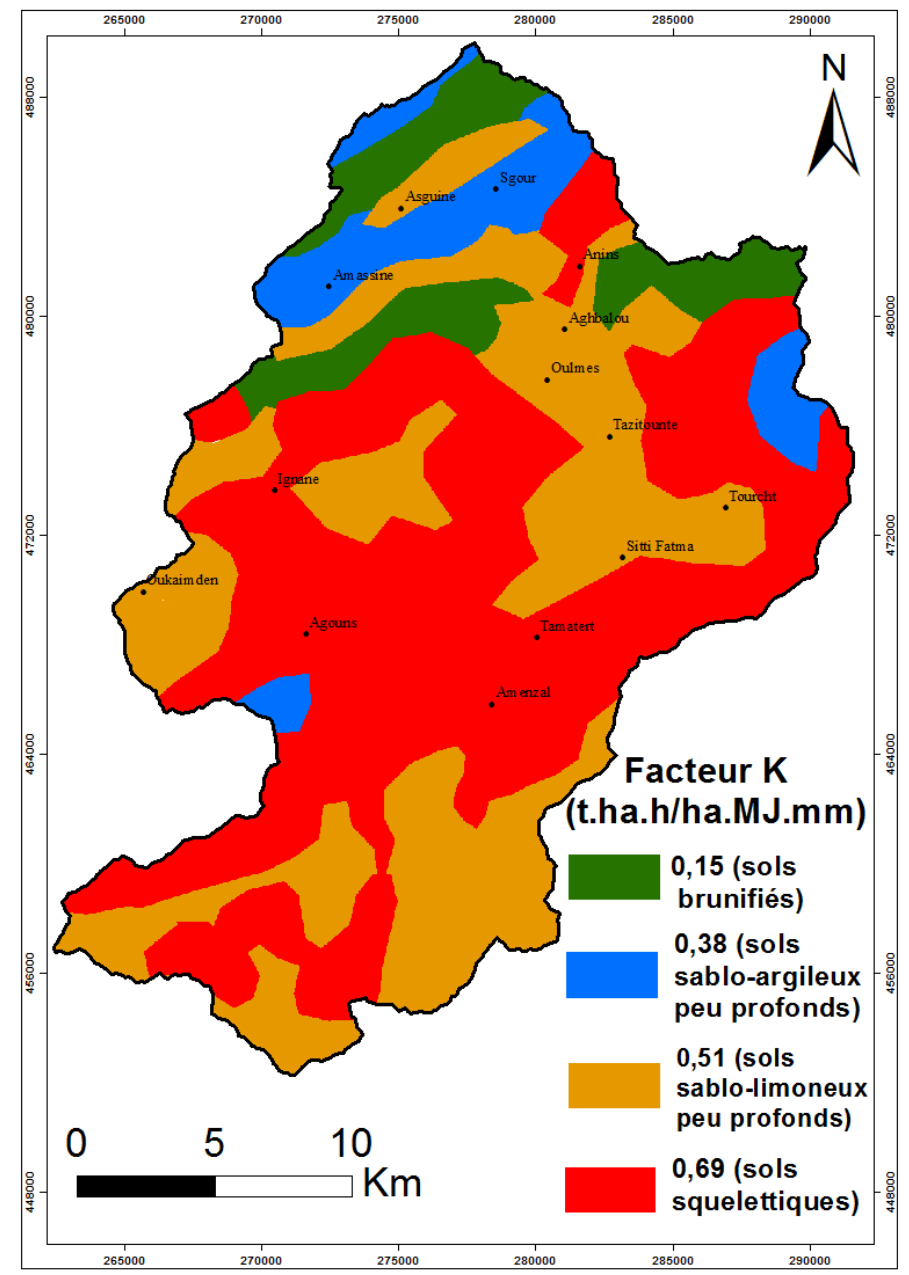

Fig 3. Carte de d'érodibilité des sols (K) dans le bassin versant de l’Ourika

Le facteur $\mathrm{K}$ dans le bassin versant de l'Ourika varie de 0,15 t.ha.h/ha.MJ.mm pour les sols les moins érodibles à 0,69 t.ha.h/ha.MJ.mm pour les sols les moins résistants à l'érosion (Fig 3). Les sols du bassin sont d'une grande sensibilité à l'érosion en ce sens que plus de $83 \%$ du bassin est d’un indice d'érodibilité K supérieur à 0,50 t.ha.h/ha.MJ.mm.

\section{Facteur topographique (LS)}

Les valeurs de LS varient de 0,01 à 94,5 et ont été regroupées en quatre classes (Fig 4). La longueur et le degré d'inclinaison de la pente sont déterminants dans le processus de l'érosion. 
Dans le bassin versant de l’Ourika, les valeurs de LS sont très élevées dans les zones intrusives des affluents. L'indice LS considéré comme faible (compris entre 0 et 5) ne représente que 25\% de la superficie du bassin. Ceci correspond aux zones de faible élévation, les zones de plaine et le lit du cours d'eau. Ainsi, le bassin est sujet à un grand risque d'érosion allant de l'amont vers l'aval. Ces résultats concordent avec ceux obtenus par plusieurs auteurs dans d'autres bassins versants (Yjjou et al., 2012 ; Yjjou et al., 2014 ; Markhi et al., 2015). Il a été démontré que l'érosion croît exponentiellement en fonction du degré de pente avec un exposant moyen de 1,4 (Elbouqdaou et al., 2005). De même, il a été rapporté que lorsque le degré d'inclinaison de la pente croît, l'énergie cinétique des pluies demeure constante alors que le transport s'accélère vers le bas à cause d'une augmentation de l'énergie cinétique du ruissellement (Ibrahimi et al., 2005).

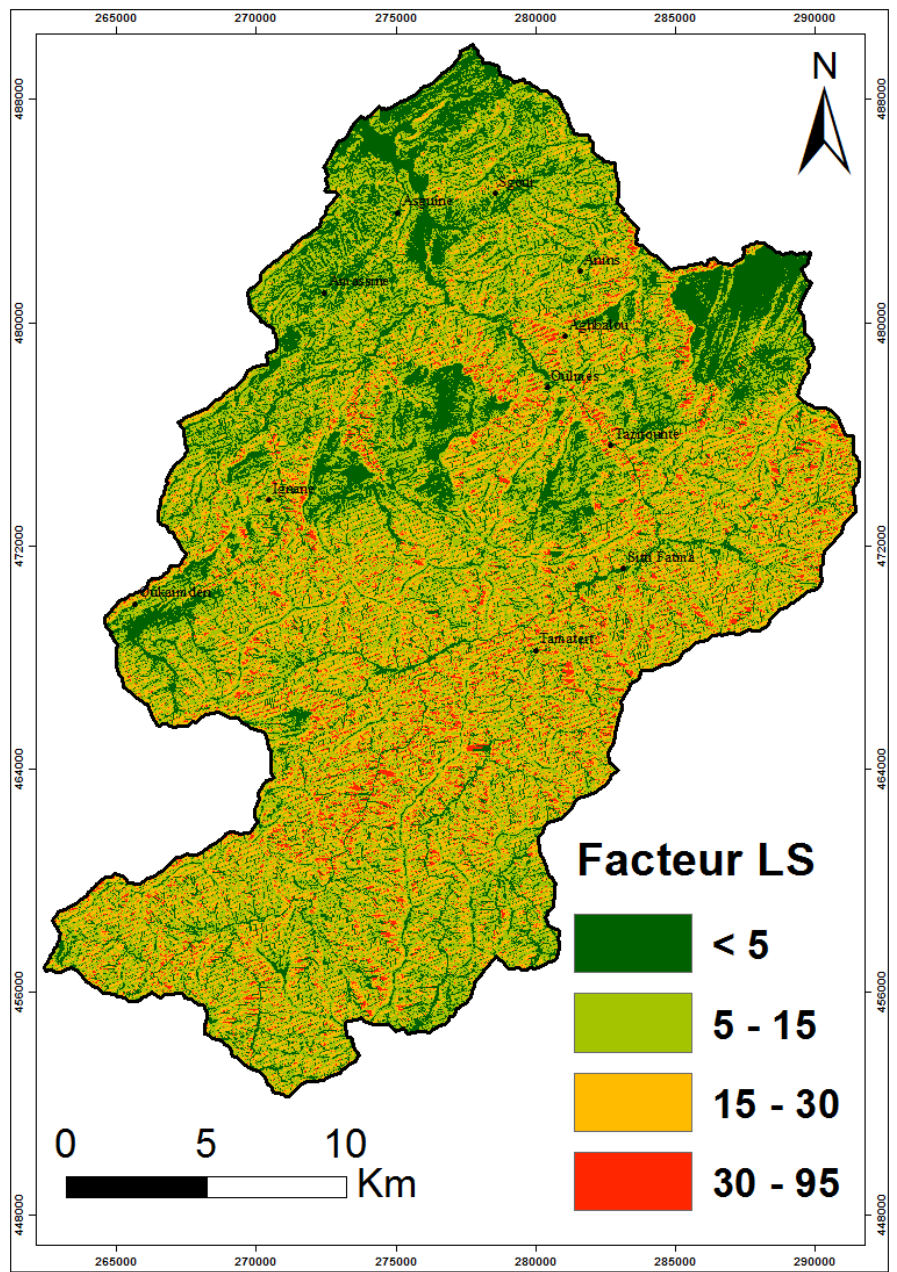

Fig 4. Carte du facteur topographique (LS) dans le bassin versant de l’Ourika 


\section{Facteur de couverture végétale $(C)$}

Le facteur $\mathrm{C}$ représente la couverture et le degré de production végétale. La carte de répartition spatiale de l'indice de couvert montre que les zones les plus sensibles à l'érosion sont les occupations du sol à faible densité de végétation qui couvrent principalement la partie amont du bassin versant (Fig 5).

Les résultats obtenus montrent que $83 \%$ de la superficie du bassin présente un taux de couvert végétal très faible et seulement $13 \%$ de la superficie est bien protégée avec $C<0,5$. Le faible couvert végétal est rencontré dans les vides asylvatiques, les forêts claires, les parcours dégradés et les terrains cultivés qui sont considérés très sensibles à l'érosion (Meliho et al., 2016). Les valeurs inférieures à 0,5 concernent les forêts denses, les matorrals denses et l'arboriculture. Les valeurs comprises entre 0,5 et 0,9 se retrouvent dans les zones couvertes par les forêts peu denses, clairsemées et les matorrals clairs. Les valeurs qui tendent vers 1 sont liées aux sols nus et aux terrains de céréaliculture récoltée. Les zones à faible couvert végétal (facteur C supérieur à 0,5) sont d'une grande sensibilité à l'érosion hydrique.

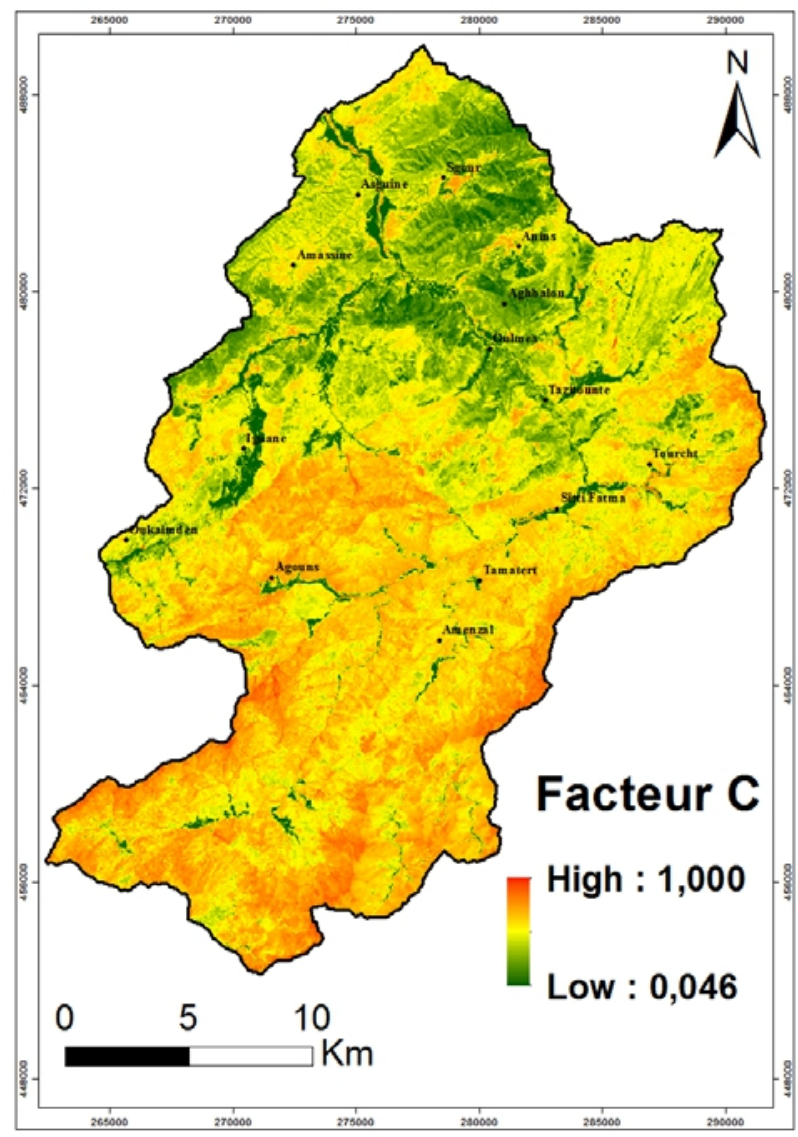

Fig 5. Carte du facteur couvert végétal (C) dans le bassin versant de l’Ourika. 


\section{Facteur des pratiques antiérosives $P$}

Les techniques culturales telles que le labour selon les courbes de niveau, les cultures en bandes alternées ou en terrasses, le buttage et le billonnage sont les pratiques efficaces de conservation des sols.

Les valeurs du facteur $\mathrm{P}$ sont inférieures ou égales à 1 . La valeur 1 correspond aux terrains sans pratiques antiérosives. Le facteur $\mathrm{P}$ varie selon la pratique agricole ou de lutte antiérosive adoptée et aussi selon la pente.

Il faut noter que dans cette étude les valeurs du facteur $\mathrm{P}$ ont été déterminées selon la pente. Les valeurs faibles et moyennes correspondent aux zones de pente faible à modérée. Les valeurs du facteur $\mathrm{P}$ varient entre 0,55 et 0,6 pour les zones à faible pente et entre 0,8 et 1 pour les zones à pentes fortes. La valeur de $\mathrm{P}$ est égale à 1 pour $50 \%$ de la superficie du bassin (Fig 6).

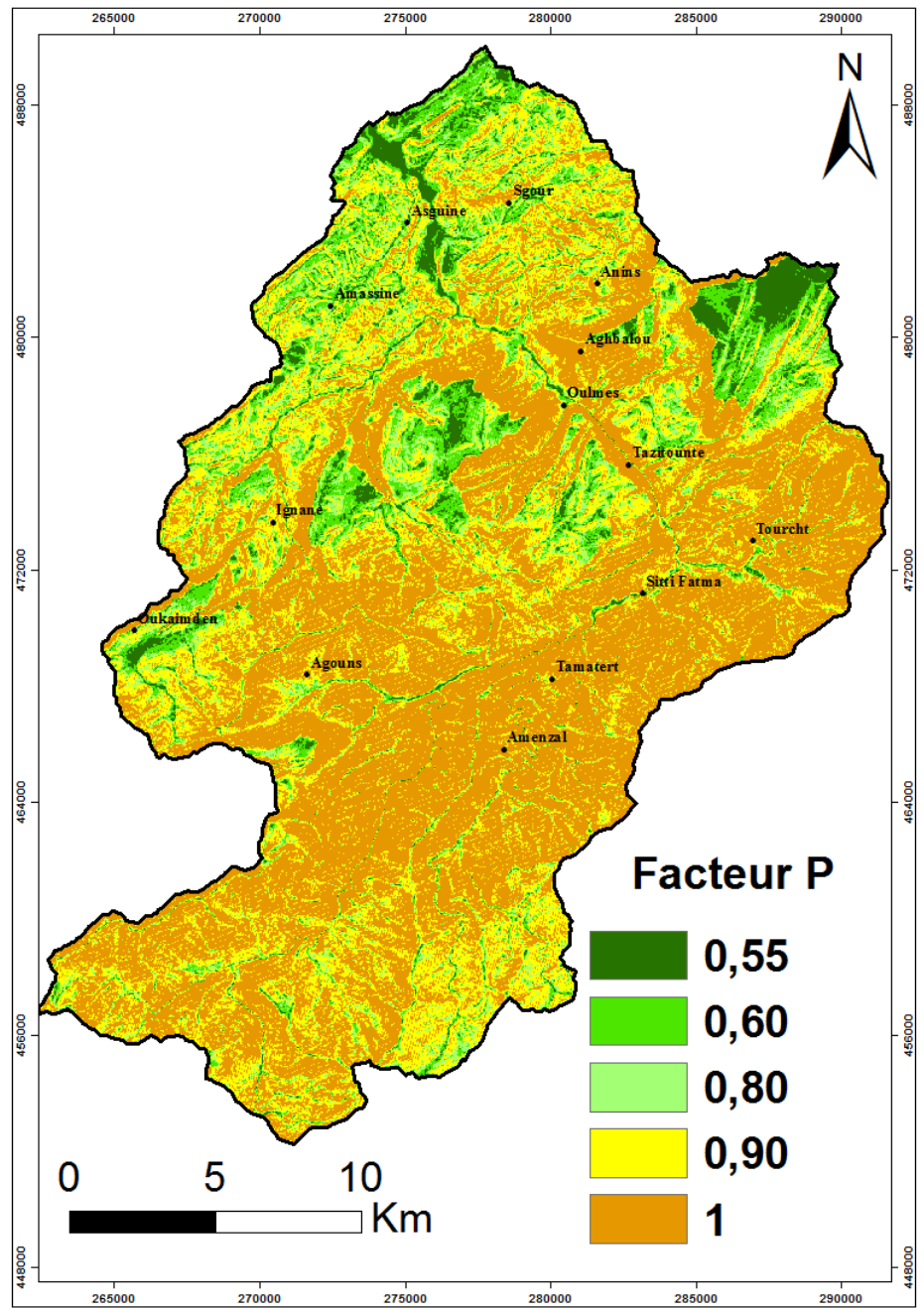

Fig 6. Carte du facteur antiérosif (P) dans le bassin versant de l’Ourika. 


\section{Evaluation des pertes en sols}

Les pertes en sols résultent de la combinaison des facteurs du modèle RUSLE, à savoir l'agressivité climatique $\mathrm{R}$, l'érodibilité des sols $\mathrm{K}$, le facteur topographique LS, le couvert végétal $\mathrm{C}$ et les pratiques antiérosives P. La combinaison des cartes de ces principaux facteurs dans un environnement SIG a permis d'obtenir la carte des pertes en sols en tout point du bassin versant.

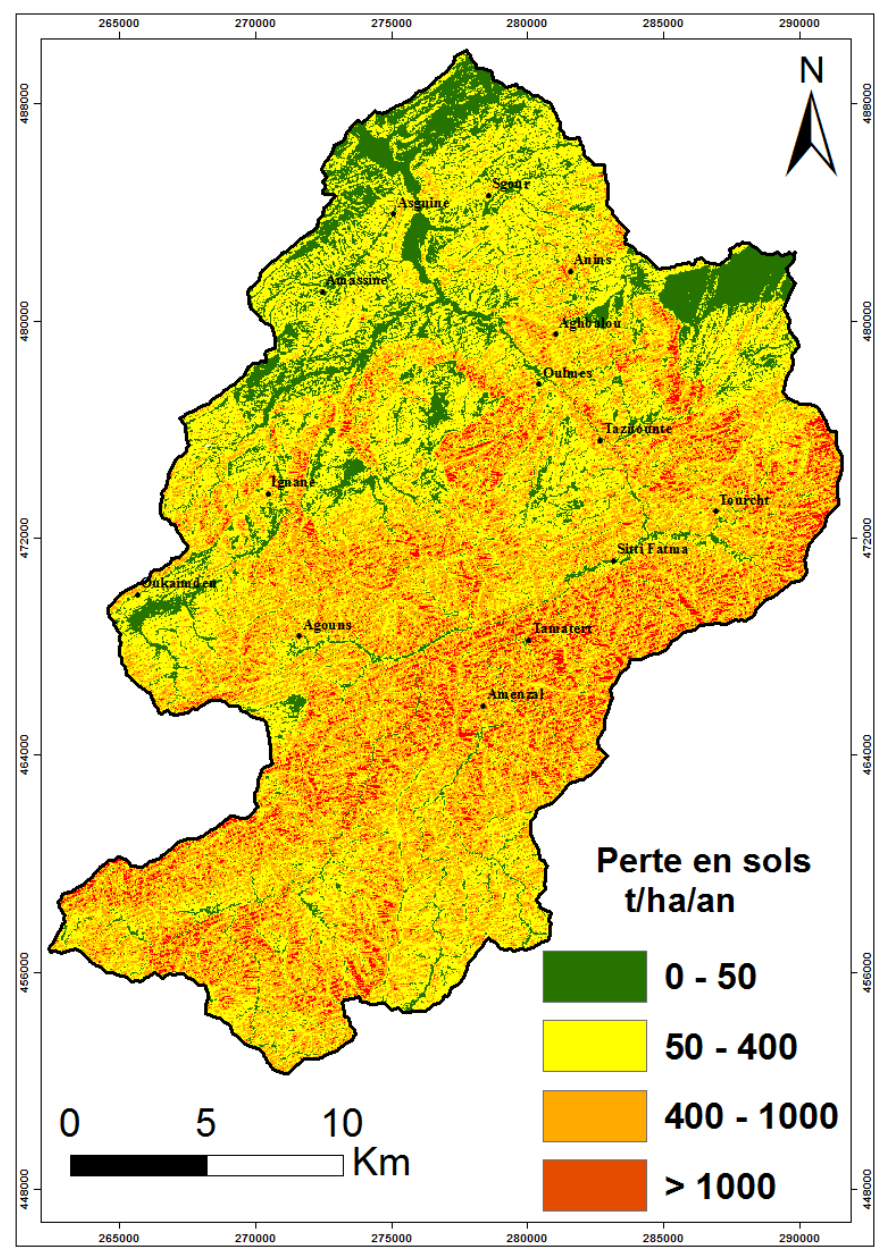

Fig 7. Carte des pertes en sols dans le bassin versant de l’Ourika

Tableau 2. Répartition des pertes en sol dans le bassin versant de l'oued Ourika.

\begin{tabular}{|c|c|c|}
\hline Pertes en sol (t/ha/an) & Surface (ha) & \% surface du bassin \\
\hline $0-50$ & 8289 & 15 \\
\hline $50-400$ & 27857 & 48 \\
\hline-1000 & 17472 & 30 \\
\hline$>1000$ & 3981 & 7 \\
\hline Total & 57600 & 100 \\
\hline
\end{tabular}


Les pertes moyennes en sols dans le bassin versant de l'Ourika sont évaluées à 380 t/ha/an avec un écart-type de 362 t/ha/an, ce qui témoigne de l'importance et de la grande variabilité du phénomène d'érosion hydrique dans le bassin. Les pertes en sols (t/ha/an) dans le bassin ont été regroupées en 4 classes de valeurs (Fig 7 et Tableau 2). La première classe concerne les zones dont la perte en sol est inférieure à 50 t/ha/an. Elle représente 15\% de la superficie du bassin et couvre principalement les zones qui se trouvent en aval du bassin versant. La deuxième classe concerne les zones dont la perte en sol est comprise entre 50 et 400 t/ha/an. Elle représente $48 \%$ de la superficie du bassin. La troisième classe concerne les zones dont la perte en sol est comprise entre 400 et 1000 t/ha/an. Elle représente $30 \%$ de la superficie du bassin. La quatrième classe regroupe les zones dont la perte en sol est supérieure à 1000 t/ha/an. Elle ne constitue que 7\% de la superficie du bassin versant. Ces deux dernières classes concernent les zones montagneuses et les zones à substrat friable localisées généralement à l'amont du bassin versant.

Ainsi, 75\% de la surface du bassin versant présente un taux d'érosion supérieur à $50 \mathrm{t} / \mathrm{ha} / \mathrm{an}$. Ce chiffre correspond à un taux très important d'érosion qui ne peut être compensé par pédogenèse. Les faibles pertes, en dessous du seuil de tolérance ( $<7 \mathrm{t} / \mathrm{ha} / \mathrm{an})$, ne concernent que $4 \%$ de la superficie du bassin versant.

Ces résultats sont proches de ceux obtenus dans des conditions similaires du bassin versant d'Oum Er-Rbia où les pertes en sol de la classe 50 - 400 t/ha/an représentent 54\% de la superficie du bassin (Yjjou et al., 2012 ; Yjjou et al., 2014).

Les résultats obtenus diffèrent légèrement de ceux rapportés pour le bassin versant de N'fis (Markhi et al., 2015), un bassin du Haut Atlas de Marrakech. En effet, la valeur maximale des pertes en sols est inférieure à $1500 \mathrm{t} / \mathrm{ha} / \mathrm{an}$ dans le bassin versant de N'fis alors qu'elle est supérieure à 2000 t/ha/an dans le bassin versant de l'Ourika. Ceci peut s'expliquer par le fait que le facteur d'érosivité des pluies et le facteur topographique sont plus importants dans le bassin de l'Ourika que dans celui de N'fis. L’érosivité des pluies atteint $100 \mathrm{MJ}$. mm/ha.h.an dans le bassin de l'Ourika alors que la valeur maximale est de $57 \mathrm{MJ}$. mm/ha.h.an dans le bassin versant de N'fis. De même, le facteur topographique est plus important dans le bassin versant de l'Ourika que dans celui de N'fis. Ainsi, bien que les deux bassins versant se trouvent dans le même contexte haut atlasique, les conditions climatiques et topographiques sont légèrement différentes, ce qui peut expliquer les différences observées dans la vulnérabilité des deux bassins à l'érosion hydrique.

L’applicabilité du modèle empirique de l'équation universelle des pertes en sols dans des conditions différentes de celles où il a été élaboré 
l'exposent à de nombreuses critiques en plus du fait qu'il ne s'applique qu'à l'érosion en nappe (Renard et al., 1997). De plus, le modèle considère que toutes les surfaces sont soumises à l'érosion si tous les facteurs sont non nuls et ne met pas en évidence les dépôts. Cependant, l'incertitude reste tolérable si les mesures de terrain et les analyses de laboratoire sont rigoureusement effectuées (Renard et al., 1997).

L'intégration du modèle dans un SIG présente beaucoup d'avantages. Elle permet de gérer d'une façon efficace une quantité importante de données relatives aux différents facteurs de l'érosion hydrique. Elle permet aussi établir une carte synthétique des pertes en sol ou de l'érosion potentielle ( $\mathrm{t} / \mathrm{ha} / \mathrm{an})$ et de répartition spatiale de la vulnérabilité à l'érosion des différentes zones du bassin versant. Il faudrait mentionner que l'équation universelle des pertes en sols n'évalue que les pertes moyennes causées par l'érosion en nappe. Le modèle est basé sur des données parcellaires ou des bassins versants de très petite surface, ce qui pose des problèmes de précision lorsqu'on l'utilise sur une plus grande échelle et dans conditions autres que celles dans lesquelles il a été adopté. Ainsi, malgré le fait que la validité des résultats obtenus est sujette à des discussions, la méthode permet aux décideurs et aux gestionnaires de planifier les interventions de lutte contre l'érosion dans les zones où le risque d'érosion est élevé. Elle permet aussi de raisonner l'utilisation des sols par des aménagements dans le but de lutter contre les phénomènes d'érosion.

\section{Conclusion}

L'étude des risques d'érosion dans le bassin versant de l'Ourika a été effectuée à l'aide de l'Equation Universelle Révisée des Pertes en Sol (RUSLE). Les différents facteurs intervenant dans les processus d'érosion ont été déterminés et leur combinaison dans un environnement SIG a permis d'obtenir les pertes en sol dans le bassin. Les résultats obtenus montrent que le bassin est soumis à une forte agressivité climatique dans sa grande partie et une forte vulnérabilité des sols à l'érosion hydrique. La valeur moyenne des pertes en sols (t/ha/an) obtenue dans le bassin est de $380 \mathrm{t} / \mathrm{ha} / \mathrm{an}$, avec $48 \%$ de la superficie du bassin soumis à des pertes en sol comprises entre 50$400 \mathrm{t} / \mathrm{ha} / \mathrm{an}$ et $30 \%$ à des pertes variant de 400 à $1000 \mathrm{t} / \mathrm{ha} / \mathrm{an}$. Les pertes en sols au-dessous du seuil de tolérance ( $<7 \mathrm{t} / \mathrm{ha} / \mathrm{an})$ ne représentent que $4 \%$ de la superficie du bassin versant. Cependant les résultats obtenus sont d'un grand intérêt pour le gestionnaire dans l'orientation des actions de lutte contre l'érosion.

\section{Remerciements :}

cette étude a été réalisée avec l'appui financier du Centre de Recherches pour le Développement International (CRDI), Canada. 


\section{References:}

1. Angima, S.D., Stott, D.E., O’Neill, M.K., Ong, C.K., Weesies, G.A. (2003). Soil erosion prediction using RUSLE for central Kenyan highland conditions. Agriculture, Ecosystems and Environment, 97 (1-3), pp. 295-308.

2. Arnalds, O., Dorarinsdottir, E.F., Metusalemsson, S., Jonsson, A., Gretarsson, E., Arnason, A. (2001). Soil Erosion in Iceland. Soil Conservation Services and Agricultural Research Institute. Iceland.

3. Arnoldus, H. M. J. (1980). Methodologie used to determine the maximum potentiel average soil loss due to sheet and rill erosion in Morrocco, Bulletin F.A.O., 34p.

4. Biron, P. E. (1982). Le Permo-Trias de la région de l'Ourika (HautAtlas de Marrakech, Maroc). Thèse de 3ème Cycle, Université de Grenoble, 170 p.

5. Boggs, G., Devonport, C., Evans, K., Puig, P. (2001). GIS-based rapid assessment of erosion risk in a small catchment in the wet/dry tropics of Australia. Land Degradation \& Development 12: 417-434.

6. Bonilla, C.A., Reyes, J.L., Magri, A. (2010). Water erosion prediction using the Revised Universal Soil Loss Equation (RUSLE) in a GIS framework, central Chile. Chilean Journal of Agricultural Research 70 (1), 159-169.

7. Boudhar, A., Duchemin, B., Hanich, L., Chaponnière, A., Maisongrande, P., Boulet, G., Stitou, J. and Chehbouni, A. (2007). Analyse de la dynamique des surfaces enneigées du Haut Atlas Marocain à partir des données SPOTVEGETATION. Sécheresse 18 (4) : 278-88.

8. Dabral, P.P., Baithuri, N., Pandey, A. (2008). Soil erosion assessment in a hilly catchment of North Eastern India using USLE, GIS and remote sensing. Water Resources Management 22, 1783-1798.

9. De Graaf, J. (1996). Price of soil erosion: an economic evaluation of soil conservation and watershed development, Landbouwuniversiteit Wageningen (LUW), Wageningen, $300 \mathrm{pp}$.

10. Del Mar López, T., Mitchel Aide, T., Scatena, F. N. (1998). The effect of land use on soil erosion in the Guadiana watershed in Puerto Rico. Caribbean Journal of Science, 34: 298-307.

11. Desmet, P. J. J., Govers, G. (1996). A GIS-procedure for automatically calculating the USLE LS-factor on topographically complex landscape units. Journal of Soil and Water Conservation, v. 51, n. 5, p. 427-433.

12. Elbouqdaou, K., Ezzine, H., Badrahou, M., Rouchdi, M., Zahraoui, M. and Ozer, A. (2005). Approche méthodologique par télédétection et SIG de l'évaluation du risque potentiel d'érosion hydrique dans le 
bassin versant de l'Oued Srou (Moyen Atlas, Maroc), Geo-Eco-Trop, 29, 25-36.

13. Hoyos, N. (2005). Spatial modeling of soil erosion potential in a tropical watershed of the Colombian Andes. CATENA 63 (1), 85-108.

14. Hudson, N. (1981). Soil Conservation. Second Edition. Comell University Press, Ithaca, New York.

15. Ibrahimi, S. (2005). Application du 210Pbexe comme une alternative à l'utilisation du 137Cs pour l'étude de la redistribution du sol sur des transects cultivés et non cultivés, Bassins versants El Hachef et Raouz, nord du Maroc, Thèse de doctorat en Sciences, Université Abdelmalek Essaadi, Tanger, Maroc.

16. Jasrotia, A.S., Singh, R. (2006). Modeling runoff and soil erosion in a catchment area, using the GIS, in the Himalayan region, India. Environmental Geology 51, 29-37.

17. Jensen, J. R. (2000). Remote sensing of the environment: An earth resource perspective, published as 1st ed. Upper Saddel River, New Jersey: Prentice Hall, Inc.

18. Kalman, R. (1967). Le facteur climatique de l’érosion dans le bassin de Sebou. Projet Sebou, Rapp. Ronéo, 40p.

19. Karydas, C.G., Sekuloska, T., Silleos, G.N. (2009). Quantification and site-specification of the support practice factor when mapping soil erosion risk associated with olive plantations in the Mediterranean island of Crete. Environmental Monitoring and Assessment 149, 19-28.

20. Kouli, M., Soupios, P., Vallianatos, F. (2009). Soil erosion prediction using the Revised Universal Soil Loss Equation (RUSLE) in a GIS framework, Chania, Northwestern Crete, Greece. Environmental Geology 57, 483-497.

21. Krishna Bahadur, K.C. (2009). Mapping soil erosion susceptibility using remote sensing and GIS: a case of the Upper Nam Wa Watershed, Nan Province, Thailand. Environmental Geology 57, 695705.

22. Lal, R. (1998). Soil erosion impact on agronomic productivity and environment quality: critical reviews. Plant Sci 17:319-464.

23. Lu, D., Li, G., Valladares, G.S., Batistella, M. (2004). Mapping soil erosion risk in Rondonia, Brazilian Amazonia: using RUSLE, remote sensing and GIS. Land Degradation and Development 15, 499-512.

24. Markhi, A., Laftouhi, N., Soulaimani, A., Fniguire, F. (2015). Quantification et évaluation de l'érosion hydrique en utilisant le modèle RUSLE et déposition intégrés dans un SIG. Application dans le bassin versant n'fis dans le haut atlas de Marrakech (Maroc). European Scientific Journal October 2015 edition vol.11, No.29. 
25. McCool, D. K., Brown, L. C., Foster, G. R. (1987). Revised slope steepness factor for the Universal Soil Loss Equation. Transactions of the ASAE, v. 30, n. 5, p. 1387-1396.

26. Meliho, M., Khattabi, A., Zine El Abidine, A. (2016). Etude de la sensibilité à l'érosion hydrique dans le bassin versant de l'Ourika (Haut Atlas, Maroc). First AMRS Congress and 23rd APDR Congress 'Sustainability of Territories in the Context of Global Changes, pp: 189-196.

27. Merzouk, A. and Dahman, H. (1998). Shilling land use and impact on sediment yield in the Rif Mountains. In advanced in geoecologique, 31, by CATENA VERLAG, 350447, Reiskirche, 1998.

28. Millward, A.A., Mersey, J.E. (1999). Adapting the RUSLE to model soil erosion potential in a mountainous tropical watershed. CATENA 38 (2), 109-129.

29. Molnar, D.; Julien, P. (1998). Estimation of upland erosion using GIS. Comput. Geosci, 24, 183-192.

30. Ouhammou A. (1986). Recherche sur l'étagement de la végétation dans le versant de l'oued Ourika (Haut Atlas Central, Maroc). Th. 3e cycle. Univ. Cadi Ayyad, Fac. Sci. Marrakech: 181p.

31. Panagos, P., Borrelli, P., Meusburger, K. (2015). A new European slope length and steepness factor (LS-Factor) for modeling soil erosion by water. Geosciences 5, 117-126.

32. Pandey, A., Mathur, A., Mishra, S.K., Mal, B.C. (2009). Soil erosion modeling of a Himalayan watershed using RS and GIS. Environmental Earth Sciences 59 (2), 399-410.

33. Prasannakumar, V., Vijith, H., Abinod, S. and Geetha, N. (2012). Estimation of Soil Erosion Risk within a Small Mountainous SubWatershed in Kerala, India, Using Revised Universal Soil Loss Equation (RUSLE) and Geo Information Technology. Geoscience Frontiers, Vol. 3, No.2, pp.209-215.

34. Rahhou M., 1999. L’érosion dans le Prérif central, zone interfluviale Leben-Sebou-Ouergha, un prolongement de l'évolution naturelle, une production sociale, Thèse doctorat d’Etat, Université Mohammed V, Rabat, 300p.

35. Rango, A. and Arnoldus, H.M.J. (1987). Aménagement des bassins versants. In: Cahiers techniques de la FAO: 1-11.

36. Renard, K.G., Foster, G.R., Weesies, G.A., McCool, D.K., Yoder, D.C. (1997). Predicting soil erosion by water-a guide to conservation planning with the Revised Universal Soil Loss Equation (RUSLE). United States Department of Agriculture, Agricultural Research Service (USDA-ARS) Handbook No. 703. 
37. Sadiki, A., Bouhlassa, S., Auajjar, J., Faleh, A. and Macaire, J. (2004). Utilisation d'un SIG pour l'évaluation et la cartographie des risques d'érosion par l'Equation universelle des pertes en sol dans le Rif oriental (Maroc) : cas du bassin versant de l’oued Boussouab, Bulletin de l'Institut Scientifique, Rabat, section Sciences de la Terre, 26, 2004, 69-79.

38. Shin, G. J. (1999). The analysis of soil erosion analysis in watershed using GIS”, Ph.D. Dissertation, Department of Civil Engineering, Gang-won National University.

39. Tian, Y.C., Zhou, Y.M., Wu, B.F., Zhou, W.F. (2009). Risk Assessment of Water Soil Erosion in Upper Basin of Miyun Reservoir, vol. 57. Environmental Geology, Beijing, China, pp. 937942.

40. Van der Knijff, J.M., Jones, R.J.A. and Montanarella, L. (2000). Soil Erosion Risk Assessment in Europe, EUR 19044 EN, 34p.

41. Vezena, K. et Bonn, F. (2006). Modélisation et analyse de la dynamique spatio-temporelle des relations société - érosion et pollution diffuse en milieu agricole - étude de cas en Vietnam et au Québec ; Interaction Nature-Société, analyse et modèles. UMR6554 LETG, LaBaule, 6p.

42. Wachal, D.J. (2007). Integrating GIS and erosion modeling - A tool for watershed management, ESRI international user conference, Paper $N^{\circ}$ UC1038, 11p.

43. Wang, G., Gertner, G., Fang, S., Anderson, A. B. (2003). Mapping multiple variables for predicting soil loss by geostatistical methods with TM images and a slope map. Photogrammetric Engineering and Remote Sensing 69: 889-898.

44. Wilson J. P., Lorang M.S. (2000). Spatial models of soil erosion and GIS. In Spatial Models and GIS: New Potential and New Models, Fotheringham AS, Wegener M (eds). Taylor \& Francis: Philadelphia, PA; 83-108.

45. Wischmeier, W.H., Smith, D.D. (1978). Predicting rainfall erosion losses. A Guide to conservation planning. United States Department of Agriculture, Agricultural Research Service (USDA-ARS) Handbook No. 537. United States Government Printing Office, Washington, DC.

46. Yjjou, M., Bouabid, R., El Hmaidi, A., Essahlaoui, A. et El Abassi, M. (2014). Modélisation de l'érosion hydrique via les SIG et l'équation universelle des pertes en sol au niveau du bassin versant de l'Oum Er-Rbia. The International Journal Of Engineering And Science (IJES), Volume 3, Issue 8, Pages, pp: 83-91. 
47. Yjjou, M., Bouabid, R., El Hmaidi, A. et Essahlaoui, A. (2012). Caractérisation topographique et climatique via le SIG du bassin versant du haut Oum Er-Rbia en amont du barrage El Hansali (SW du Moyen Atlas, Maroc), Journal of Hydrocarbons Mines and Environmental Research, 3, 2, 104-109.

48. Zhang, H., Yang, Q., Li, R., Liu, Q., Moore, D., He, P., Ritsema, C. J. \& Geissen, V. (2013). Extension of a GIS procedure for calculating the RUSLE equation LS factor. Comput. Geosci., 52, 177-188. http://dx.doi.org/10.1016/j.cageo.2012.09.027.

49. Zhou, P., Luukkanen, O., Tokola, T., Nieminen, J. (2008). Effect of vegetation cover on soil erosion in a mountainous watershed. CATENA 75 (3), 319-325. 